\title{
Spotlight on nilotinib in the treatment of chronic myelogenous leukemia
}

This article was published in the following Dove Press journal:

Blood and Lymphatic Cancer:Targets and Therapy

2I August 2014

Number of times this article has been viewed

\author{
Stephen Harnicar \\ Sherry Mathew \\ Department of Pharmacy, Memorial \\ Sloan Kettering Cancer Center, \\ New York, NY, USA
}

\begin{abstract}
Nowhere has targeted therapy been more successful in the hematologic malignancy arena than chronic myelogenous leukemia (CML). By targeting the $B C R-A B L$ fusion oncogene, the introduction of tyrosine-kinase inhibitors (TKIs) has dramatically improved the outcomes of this disease. Nilotinib is a second-generation TKI that initially gained approval for the treatment of imatinib-resistant or -intolerant disease for patients with chronic or accelerated-phase CML. Investigation in the first-line setting also demonstrated efficacy, and expanded nilotinib's approval to include therapy for patients with treatment-naïve chronic-phase CML. Data also exist for blast-phase disease, which allows nilotinib to be an option for all phases. Nilotinib's place in therapy is continuously being expanded by research in novel areas, such as post-hematopoietic stem cell transplants for prevention of relapse and in the pediatric arena. With multiple TKIs now approved for the treatment of CML, delineating the pharmacologic distinctions of nilotinib is an asset when determining therapy. By understanding the pharmacokinetics and dependence on hepatic metabolism of nilotinib, the clinician can manage the potential toxicities, interactions, and unique dosing of this drug. The recognition of mechanisms of resistance, patient adherence, and cost-effectiveness are similarly significant considerations. Actively integrating these various specifics will allow clinicians to optimize nilotinib therapy for the CML patient.
\end{abstract}

Keywords: nilotinib, chronic myelogenous leukemia, CML, tyrosine-kinase inhibitor, TKI

\section{Introduction}

The introduction of tyrosine-kinase inhibitors (TKIs) into the treatment of chronic myeloid leukemia (CML) has completely altered the landscape of this once life-threatening malignancy. Thirteen years after the US Food and Drug Administration (FDA) approval of the first-generation TKI imatinib, there are an additional four TKIs added to our arsenal in the treatment of CML. Understanding the place in therapy for the various agents and the clinical nuances between them is key to optimizing a patient's clinical outcome. Nilotinib is a second-generation TKI that has had an evolving role in the treatment of CML, and the specifics of its clinical impact and pharmacologic distinctions are briefly described here.

\section{Nilotinib pharmacodynamics and pharmacokinetics}

Nilotinib is an oral second-generation TKI and one of three in this class categorized with dasatinib and bosutinib. The translocation of $A B L$ to the $B C R$ gene, known as the Philadelphia chromosome (Ph), is the hallmark of the pathogenesis of CML. Nilotinib inhibits BCR-ABL, PDGFR, and c-Kit kinases; it is by competitively inhibiting the BCR-ABL adenosine triphosphate (ATP)-binding site that nilotinib is able to 
prevent tyrosine phosphorylation of downstream intracellular signal-transduction proteins. This in turn prevents cell proliferation and induces apoptosis of $\mathrm{Ph}^{+}$cells. Nilotinib is unique in that its potency is increased by a factor of 30 over imatinib due to its amplified ABL kinase selectivity and binding site affinity.

Nilotinib is administered twice daily orally. The bioavailability of nilotinib is significantly affected by food intake. Systemic exposure is increased $82 \%$ when taken 30 minutes prior to a high-fat meal compared with a fasted state. ${ }^{1}$ Gastric $\mathrm{pH}$ also can affect nilotinib's absorption. An area under the curve (AUC) decrease of $34 \%$ can be seen when nilotinib is taken with drugs that elevate gastric $\mathrm{pH} .{ }^{1}$ Once absorbed, nilotinib is $98 \%$ serum protein-bound. It is primarily metabolized via oxidation and hydroxylation, and continues to a second phase of metabolism via the hepatic enzyme cytochrome P450 3A4 (CYP3A4). Nilotinib is eliminated in the feces, and has an approximate half-life of 17 hours. ${ }^{1}$ Changes in renal function do not require any dose adjustments; however, no clinical studies have been performed in patients with impaired renal function. Because of nilotinib's hepatic metabolism, caution is recommended when administering nilotinib to patients with hepatic impairment. On average, higher systemic exposure after a $200 \mathrm{mg}$ nilotinib dose of $18 \%$ and $23 \%$ in patients with Child-Pugh A and B impairment, respectively, were seen. ${ }^{2}$ In another study, patients with severe Child-Pugh class $\mathrm{C}$ impairment, had a mean increase in AUC of $56 \%{ }^{1}$

\section{Nilotinib in the refractory/resistant setting}

Initial investigation of nilotinib's role in the clinical setting was characterized in a Phase I dose-escalation study for imatinib resistant patients. Nilotinib was determined to be active in imatinib-resistant CML in this setting. ${ }^{3}$ Nilotinib continued to be assessed in the imatinib-resistant or -intolerant setting in two open-label, Phase II clinical trials based on the activity seen in Phase I studies. The Phase II clinical trials were twofold, examining nilotinib in specific disease phases: one trial examined nilotinib in chronic-phase disease, and the other in accelerated-phase disease. ${ }^{4,5}$

In the chronic-phase trial, 280 patients were given nilotinib at a dose of $400 \mathrm{mg}$ twice daily. The rate of major cytogenetic response (MCyR) at 6 months was $48 \%$, and complete CyR (CCyR) was 31\%. The maintenance of a MCyR was noted in a 24-month follow-up in which $77 \%$ of responding patients were able to keep their response. Overall survival was $87 \%$ for the entire patient population. ${ }^{6}$ In the 48 -month follow-up, the overall survival rate was $78 \%$, demonstrating nilotinib's sustained efficacy. ${ }^{?}$

In the accelerated-phase trial, nilotinib was also given at a dose of $400 \mathrm{mg}$ twice daily. An MCyR was observed in 29\% of the 119 patients enrolled. Overall survival at 12 months was $79 \%$. Because of these findings, nilotinib gained FDA approval in 2007 for CML patients with imatinib-refractory or -intolerant disease in the chronic or accelerated phase.

Nilotinib has also shown promise in blast-phase disease. ${ }^{8,9}$ A complete hematologic response rate of $13 \%$ and a rate of return to chronic phase of $18 \%$ in 96 patients resistant or intolerant to imatinib were observed at the $400 \mathrm{mg}$ twice-daily dose. Despite evidence of activity in this setting, nilotinib is not yet approved by the FDA for this indication.

Nilotinib can also be used after treatment failure with two different TKIs, lending itself to a third-line indication. ${ }^{10,11}$ In a study where 14 patients received nilotinib after imatinib and dasatinib, a complete hematologic response was seen in $67 \%$, $100 \%$, and $67 \%$ of those patients with chronic phase, accelerated phase, and blast phase, respectively. A major molecular response (MMR) was observed in 33\% of those in chronic phase, though no patient obtained a MMR in accelerated- or blast-phase disease. None of the study patients discontinued nilotinib due to toxicity. These data show that especially in the chronic-phase setting, nilotinib remains a viable option after failure of two TKIs.

\section{Nilotinib in the first-line setting}

With clear evidence of nilotinib's efficacy in the setting of refractory or resistant disease, the potential role of this agent in the first-line setting was subsequently explored. A Phase II single-center trial was the first to study nilotinib in the first-line setting. At 24 months of follow-up time, 93\% of patients treated with nilotinib $400 \mathrm{mg}$ twice daily were able to obtain a CCyR. ${ }^{12}$ Nilotinib was also compared to imatinib as first-line treatment in a Phase III trial performed in 846 newly diagnosed patients, titled ENESTnd (Evaluating Nilotinib Efficacy and Safety in clinical Trials - newly diagnosed patients). ${ }^{13}$ Two doses of nilotinib were compared: $300 \mathrm{mg}$ twice a day and $400 \mathrm{mg}$ twice a day. The rates of MMR for both the 300 and $400 \mathrm{mg}$ regimens were nearly double those of imatinib at a time point of 12 months. The rates of CCyR by 12 months were significantly higher for both doses of nilotinib than for imatinib. There was also a significant improvement seen in the time to progression to the accelerated or blast phase in the nilotinib arms compared with the imatinib arm. In June 2010, the FDA granted approval of nilotinib $300 \mathrm{mg}$ twice a day in newly diagnosed chronic-phase CML 
based on results of ENESTnd. Subsequently, 1- and 3-year follow-up data have been published that also favored nilotinib in terms of MMR, complete molecular response, and fewer progressions to accelerated or blast phase. ${ }^{14,15}$

Even with robust and durable responses, the overall survival impact of faster and deeper molecular responses is yet to be solidified. ${ }^{16}$ There are data, however, that early molecular responses (EMRs) with nilotinib may provide some long-term benefit. Hughes et al explored the impact of EMR on outcomes in the ENESTnd trial based on a 4-year follow-up period. ${ }^{17}$ Results showed that fewer patients had EMR failure with nilotinib than imatinib, which led to higher rates of molecular response, a decrease in risk of progression, and higher overall survival.

\section{Nilotinib after allogeneic transplant}

Prior to TKIs, hematopoietic stem cell transplant (HSCT) was at one time the standard of care for CML patients if a human leukocyte antigen match was found. Now, HSCT is reserved for those who do not respond or show progressive disease to first-line therapy with TKIs. It is also a viable option for those with the T315I mutation and those that present in blast phase. ${ }^{18}$ Post-HSCT, these poor-risk patients are at risk for relapse (nearly $40 \%$ in the blast phase) which invites room for improvement in this area. ${ }^{19}$

There are data that show imatinib can play a role in the posttransplant setting to prevent relapse. ${ }^{20}$ There are fewer data however for second-generation TKIs in this setting. Dasatinib has been described in a series of nine patients with blast-phase disease. ${ }^{21}$ In the same report, nilotinib was used in two patients with $\mathrm{Ph}^{+}$acute lymphoblastic leukemia postHSCT. One patient tolerated the treatment with $400 \mathrm{mg}$ twice daily of nilotinib; however, the second stopped prophylaxis due to gastrointestinal intolerance. Data are still lacking post-HSCT with nilotinib in the CML setting.

\section{Nilotinib in the pediatric setting}

CML is extremely rare in children, and accounts for less than $5 \%$ of all pediatric leukemias. Allogeneic transplant has historically been the gold standard of care for pediatric CML. However, with the efficacy of TKIs clearly evidenced in the treatment of adult CML, a similar shift toward TKIs as first-line therapy for pediatric CML followed. ${ }^{22}$ Imatinib has been the most thoroughly investigated TKI in pediatric CML, with nilotinib therapy limited. ${ }^{23,24}$ Despite the paucity of information, understanding the emerging data regarding the use of TKIs in children can provide insight to possible pediatric considerations with this agent.
Results from pediatric patients enrolled in nilotinib's "compassionate use" program in $\mathrm{Ph}^{+} \mathrm{CML}$ and acute lymphoblastic leukemia showed a safety profile similar to that in adults. Dosing was dichotomized by weight, where patients less than $40 \mathrm{~kg}$ received $300 \mathrm{mg}$ twice daily, while patients greater than $40 \mathrm{~kg}$ received the adult dose. Nine of the sixteen patients in this resistant or refractory population showed signs of clinical response, and reported adverse effects were similar to those in adults, with a reported grade 4 neutropenia, grade 3 transaminitis, and one death from disease progression. ${ }^{25}$ A Phase I pharmacokinetic study is under way to establish pediatric dosing for nilotinib in both the newly diagnosed and refractory/resistant CML setting. The study is evaluating the safety and tolerability of nilotinib in children dosed at 230 $\mathrm{mg} / \mathrm{m}^{2}$ twice daily for up to 24 cycles. $^{26}$

Although only reported to date with imatinib use in children, growth failure is an important unique toxicity to consider when using TKIs in pediatric patients. It is hypothesized that there is a disruption in the growth hormone (GH): insulin-like growth factor-1 (IGF-1) axis, but the exact mechanism is yet to be clearly identified. Interruption in the signaling cascade may result in IGF-1 deficiency or alternatively there is direct inhibition at IGF-1 receptors with TKIs. ${ }^{27}$ An animal model testing the effects of imatinib, dasatinib, and bosutinib showed similar IGF-1 deficiencies and found insulin-like growth factor-binding protein 3 (IGFBP-3) levels were significantly lower than controls for all three TKIs. ${ }^{28}$ Children on nilotinib should be carefully monitored for signs of growth failure, and testing for serum IGF-1 may be warranted if observed. Management of this toxicity with GH therapies has not yet been investigated, although future investigation may be forthcoming. ${ }^{27}$

\section{Clinical use of nilotinib (interactions and toxicities)}

In all CML settings, drug interactions are of concern when initiating nilotinib therapy. As mentioned previously, drugs that increase gastric pH can decrease nilotinib's bioavailability. Proton-pump inhibitors (PPIs) suppress gastric acid secretion by inhibiting the parietal cell hydrogen potassium ATPase pump, which results in an eventual elevated gastric $\mathrm{pH}$. Since the effect of PPIs is long-lasting, a separation in administration times of nilotinib and PPIs or increase in nilotinib dose is unlikely to be of benefit. Coadministration is not recommended for this reason. ${ }^{1}$ Alternatives may include histamine antagonists or aluminum/magnesium-containing antacids. Histamine antagonists can be administered 10 hours 
after or 2 hours prior to a nilotinib dose, whereas antacids can be given 2 hours prior or 2 hours postdose. ${ }^{1}$

Other interactions are relevant, because nilotinib is metabolized in the liver, specifically through the CYP3A4 enzyme pathway. Therefore, caution should be used in patients that are on concomitant medications known to be strong inhibitors and inducers of CYP3A4, and when necessary appropriate medication adjustments should be made. ${ }^{29}$ Ketoconazole is a potent CYP3A4 inhibitor that has been shown to increase nilotinib exposure threefold; conversely, when the potent CYP3A4 inducer rifampin is given, systemic exposure is decreased nearly $80 \% .{ }^{1}$ Due to concerns of increased toxicity or decreased effectiveness, concomitant administration with these and similar drugs should be avoided. In current clinical practice, later-generation azoles (eg, voriconazole and posaconazole) are frequently used as prophylaxis due to an increased susceptibility to fungal infections in the high-risk CML population. Although no specific study exists, concomitant therapy with these strong CYP3A4 inhibitors warrants particular attention, because they have the potential to display the same interaction as ketoconazole. An effort should be made to avoid combination of these agents with nilotinib, as there are no clinical data to determine the degree of nilotinib dose adjustment. However, if concomitant administration cannot be avoided, a consideration can be made to reduce the nilotinib dose to $300 \mathrm{mg}$ once daily in patients with resistant or intolerant chronic- or accelerated-phase CML or $200 \mathrm{mg}$ once daily in patients with newly diagnosed, chronic-phase disease. Because of the concern of increasing the serum concentration of nilotinib in this situation and in turn toxicities, monitoring the QT interval for prolongation and other adverse effects is crucial to prevent patient harm. ${ }^{1}$

Among nilotinib's most concerning toxicities is QTc prolongation. Data from clinical trials have shown that an increase in QTc $>60 \mathrm{~ms}$ from baseline was observed in over $4 \%$ of patients and a QTc of $>500 \mathrm{~ms}$ was observed in $<1 \%$. ${ }^{1,30}$ The FDA has mandated a black-box warning as a result of reports of sudden death from this toxicity. Based on over 5,000 patients in multiple clinical studies, sudden deaths with nilotinib have been reported in $0.3 \%$ of CML patients. Although the incidence of this toxicity is low, the risks associated with it require that careful precautionary measures are taken. Before nilotinib initiation, clinicians should conduct a thorough medication review and be diligent in discontinuing drugs that can prolong the QTc. Replacement by appropriate alternate therapy can be initiated after an appropriate washout period. Because of the QTc-prolongation risk, an assessment of electrolytes should be undertaken prior to initiation of nilotinib and throughout therapy. Patients with electrolyte abnormalities, specifically hypokalemia and hypomagnesemia, should have these corrected if found. QTc prolongation is a concentration-dependent toxicity. Nilotinib absorption is affected by the presence of food, which can increase nilotinib exposure compared to a fasted state. This increase in exposure can increase a patient's risk of QTinterval prolongation; therefore, it is important to counsel patients to avoid food for at least 2 hours before and 1 hour after taking nilotinib. Further monitoring for this toxicity requires an electrocardiogram. This diagnostic test should be undertaken to monitor a patient's QTc at baseline. Continued monitoring is required 7 days after initiation of nilotinib and periodically thereafter. A QTc $>480 \mathrm{~ms}$ is the recommended threshold at which nilotinib should be held, and possible dose adjustments should be done. ${ }^{1}$ Because of the gravity of the risk of increase in QTc, the FDA approved a Risk Evaluation and Mitigation Strategy in March 2010 for nilotinib. The goal of this program is to help reduce medication errors involving food-drug interactions as well as incorrect dosing intervals via an updated medication guide and a clinician-patient communication plan. ${ }^{31}$

Hepatic toxicity is another concern, and monitoring is recommended to be done at least monthly; dose-adjustment guidelines exist for this toxicity, as well as for increases in serum lipase and amylase. ${ }^{1}$ Nilotinib should be held for any grade 3 or greater hepatic toxicity until a resolution to grade 1 or less, at which point once-daily dosing is recommended. The pathogenesis of hepatic toxicity is explained in that nilotinib is a potent inhibitor of UGT1A1, a hepatic metabolism pathway for small lipophilic molecules, such as bilirubin. ${ }^{32}$ A statistically significant increase in hyperbilirubinemia was associated with the (TA)7/(TA)7 genotype (UGT1A1*28) in a pharmacogenetic analysis of 97 nilotinib patients compared with the homozygous wild-type and heterozygous genotypes. ${ }^{1}$

Nilotinib is also capable of causing neutropenia and thrombocytopenia. Grade 3/4 myelosuppression can occur in up to $40 \%$ of patients on nilotinib therapy, and typically occurs in the first 2-3 months. Management strategies should be instituted if myelosuppression occurs. First, clinicians should hold the drug and monitor for resolution of myelosuppression. If myelosuppression persists for more than 2 weeks after holding nilotinib, the dose should be reduced to $400 \mathrm{mg}$ once daily regardless of the phase of disease. ${ }^{1}$ Unfortunately, if these measures do not improve myelosuppression, a patient's response to therapy may be affected. ${ }^{33}$ Dose adjustments for interactions and toxicities are outlined in Table 1. 
Table I Selected dose adjustments for nilotinib'

QTc prolongation

- EKG $>480 \mathrm{~ms}$
Hold nilotinib, and correct serum potassium and magnesium if low to normal limits. Evaluate concomitant medications.

Resume within 2 weeks at prior dose if QTc returns to $<450 \mathrm{~ms}$ and to within $20 \mathrm{~ms}$ of baseline.

If QTc is between $450 \mathrm{~ms}$ and $480 \mathrm{~ms}$ after 2 weeks, reduce the dose to $400 \mathrm{mg}$ once daily. If following dose reduction to $400 \mathrm{mg}$ once daily QTc returns to $>480 \mathrm{~ms}$, nilotinib should be discontinued.

Hold nilotinib.

Resume within 2 weeks at prior dose if ANC $>1 \times 10^{9} / \mathrm{L}$ and platelets $>50 \times 10^{9} / \mathrm{L}$. If blood counts are still low for $>2$ weeks, reduce dose to $400 \mathrm{mg}$ once daily.

Hold nilotinib.

Resume treatment at $400 \mathrm{mg}$ once daily if serum lipase or amylase returns to $\leq$ grade I.

Hold nilotinib.

Resume treatment at $400 \mathrm{mg}$ once daily if bilirubin returns to $\leq$ grade I.

Hold nilotinib.

Resume treatment at $400 \mathrm{mg}$ once daily if hepatic transaminases return to $\leq$ grade I.

Consider dose reduction to $300 \mathrm{mg}$ once daily in patients with resistant or imatinib-intolerant CML or $200 \mathrm{mg}$ once daily in first-line treatment for chronic-phase CML. inhibitors

Abbreviations: EKG, electrocardiogram; ANC, absolute neutrophil count; CYP, cytochrome P450; CML, chronic myeloid leukemia.

\section{Nilotinib and comorbidities}

The aforementioned nilotinib toxicities may be cause for a clinician to choose a different TKI in the comorbid patient, due to tolerability or the need for dose adjustments. For example, patients with hyperlipidemia and hypertension are predisposed to development of peripheral artery occlusive disease (PAOD).$^{34} \mathrm{~A}$ recent report retrospectively analyzed the incidence of PAOD in patients treated with nilotinib versus imatinib. ${ }^{35}$ The relative risk of diagnosis of asymptomatic PAOD, defined as a $<0.9$ ratio difference in systolic blood pressure between the upper and lower extremities, was 10.3 (95\% confidence interval 3.2-61.5) for first-line nilotinib versus first-line imatinib. Therefore, caution with nilotinib use should be taken in patients with preexisting risk factors for PAOD. Conversely, there are cardiac comorbidities in which nilotinib may be a preferable agent over other TKIs. Congestive heart failure and atrial fibrillation can exacerbate pleural effusions (a pertinent side effect of dasatinib), and have been shown in multivariate results of a single-institution investigation to be risk factors of pleural effusion development in patients with CML. ${ }^{36}$

Gugliotta et al rightly pointed out that many studies evaluating the association between comorbidities and TKI adverse effects are retrospective analyses, and expert opinion regarding available data suggest that there are no absolute comorbid contraindications to treat a patient with one TKI over another. ${ }^{37}$ However, the authors did say with more certainty that there are data lacking on safety in comorbid patients for dasatinib and even more so with nilotinib. Given this dearth of information, it is important for clinicians to be cognizant of patient conditions that can worsen TKI side effects, and at the same time understand that comorbidities are only one factor in selecting a TKI.

\section{Other nilotinib considerations}

Drug-resistant disease is a concern when treating CML with TKIs. Resistance can occur from disease point mutations or patient nonadherence. Point mutations can occur within any component of the BCR-ABL kinase domain: phosphate-binding (P) loop, intervening sequence, activation (A) loop, and catalytic (C) loop..$^{38}$ The T315I point mutation is of special concern, as this point mutation is resistant to all commercially available TKIs except ponatinib. Because of nilotinib's distinctive structure and fit into the active ABL kinase site, it is less predisposed to point mutations than imatinib. In spite of this, point mutations that tend to show resistance to nilotinib are Y253K, E255K, E255V, F359V, and F359C. ${ }^{39}$ It should be taken into consideration that some mutations occur more frequently in certain disease phases. Point mutations that are relevant to nilotinib occur more frequently in accelerated- or blast-phase disease: Y253, E255, and $\mathrm{T} 351 .{ }^{40}$ Currently, mutation analysis is not routinely performed upon TKI initiation, even though the possible presence of preexisting point mutations may be alarming to the clinician starting nilotinib therapy. In clinical practice, mutation analysis is typically performed only in cases in which initial responses are deficient or after disease progression. ${ }^{41}$ 
Poor patient adherence is a limiting factor with any oral therapy, and TKI therapy is no different. Imatinib data suggest an alarming amount of patients do not adhere to their treatment regimen, leading to an increase in overall health care cost. ${ }^{42}$ Adherence may be of utmost importance with nilotinib, since nilotinib dose interruption is strongly associated with EMR failure and therefore poor long-term outcomes. ${ }^{17}$ Studying patient adherence with nilotinib, as well as medical visits and costs, Wu et al retrospectively followed patients for 6 months after their first prescription of either nilotinib or dasatinib. ${ }^{43}$ A total of 452 dasatinib and 69 nilotinib patients were studied. In all end points, nilotinib fared better. Nilotinib was associated with fewer inpatient days and inpatient admissions and a lower total medical costs than dasatinib. Furthermore, nilotinib patients were more likely to adhere to the treatment regimen, which is surprising, given its twice-a-day regimen. Congruent with the previous study, a UK economic analysis evaluating incremental cost-effectiveness ratios per quality-adjusted life-year illustrated that first-line nilotinib proved to be more cost-effective than first-line imatinib. ${ }^{44}$

\section{Conclusion}

Nilotinib plays an integral part in the current management of CML, and has established its efficacy in both the firstand second-line settings. The continued evaluation of the strong responses seen in these areas will give clinicians a better understanding of nilotinib's place in therapy compared with other TKIs. As current data are limited, robust data are needed to continue to expand nilotinib's role in specific patient populations. The clinical considerations of nilotinib's toxicities and interactions with corresponding dose adjustments, modes of drug resistance, and patient adherence with associated costs are essential in maximizing its benefit, and will allow clinicians to optimize therapy and tailor treatment to each patient.

\section{Disclosure}

The authors report no conflicts of interest in this work.

\section{References}

1. Tasigna (nilotinib) [package insert]. East Hanover, NJ: Novartis Pharmaceuticals; 2014.

2. Holland J, Tanaka C, Zhou W, Sethuraman V, Heuman DM, Schran H. Pharmacokinetics of nilotinib in subjects with normal or impaired hepatic function. Blood. 2007;110:2379.

3. Kantarjian H, Giles F, Wunderle L, et al. Nilotinib in imatinib-resistant CML and Philadelphia chromosome-positive ALL. $N$ Engl $J$ Med. 2006;354:2542-2551.

4. Kantarjian HM, Giles F, Gattermann N, et al. Nilotinib (formerly AMN107), a highly selective BCR-ABL tyrosine kinase inhibitor, is effective in patients with Philadelphia chromosome-positive chronic myelogenous leukemia in chronic phase following imatinib resistance and intolerance. Blood. 2007;110:3540-3546.
5. le Coutre P, Ottmann OG, Giles F, et al. Nilotinib (formerly AMN107), a highly selective BCR-ABL tyrosine kinase inhibitor, is active in patients with imatinib-resistant or -intolerant accelerated-phase chronic myelogenous leukemia. Blood. 2008;111:1834-1839.

6. Kantarjian HM, Giles FJ, Bhalla KN, et al. Update on imatinib-resistant chronic myeloid leukemia patients in chronic phase (CML-CP) on nilotinib therapy at 24 months: clinical response, safety, and long-term outcomes. Blood. 2009;114:1129.

7. Giles FJ, le Coutre PD, Pinilla-Ibarz J, et al. Nilotinib in imatinibresistant or imatinib-intolerant patients with chronic myeloid leukemia in chronic phase: 48-month follow-up results of a phase II study. Leukemia. 2013;27:107-112.

8. Giles FJ, Kantarjian H, le Coutre PD, et al. Use of nilotinib to induce responses with 24-month (mo) minimum follow-up in patients (pts) with chronic myeloid leukemia in blast crisis (CML-BC) resistant to or intolerant of imatinib. J Clin Oncol. 2010;28:6510.

9. Ottmann O, Kantarjian H, Larson R, et al. A phase II study of nilotinib, a novel tyrosine kinase inhibitor administered to imatinib resistant or intolerant patients with chronic myelogenous leukemia (CML) in blast crisis $(\mathrm{BC})$ or relapsed/refractory $\mathrm{Ph}+$ acute lymphoblastic leukemia (ALL). Blood. 2006;108:1862.

10. Giles FJ, le Coutre P, Bhalla KN, et al. Nilotinib therapy after dasatinib failure in patients with imatinib-resistant or -intolerant chronic myeloid leukemia (CML) in chronic phase (CP), accelerated phase (AP) or blast crisis (BC). Blood. 2007;110:1029.

11. Garg RJ, Kantarjian H, O'Brien S, et al. The use of nilotinib or dasatinib after failure to 2 prior tyrosine kinase inhibitors: long-term follow-up. Blood. 2009;114:4361-4368.

12. Cortes J E, Jones D, O’Brien S, et al. Nilotinib as front-line treatment for patients with chronic myeloid leukemia in early chronic phase. J Clin Oncol. 2010;28:392-397.

13. Saglio G, Kim DW, Issaragrisil S, et al. Nilotinib versus imatinib for newly diagnosed chronic myeloid leukemia. $N$ Engl J Med. 2010;362:2251-2259.

14. Kantarjian HM, Hochhaus A, Saglio G, et al. Nilotinib versus imatinib for the treatment of patients with newly diagnosed chronic phase, Philadelphia chromosome-positive, chronic myeloid leukaemia: 24-month minimum follow-up of the phase 3 randomised ENESTnd trial. Lancet Oncol. 2011;12:841-851.

15. Larson RA, Hochhaus A, Hughes TP, et al. Nilotinib vs imatinib in patients with newly diagnosed Philadelphia chromosome-positive chronic myeloid leukemia in chronic phase: ENESTnd 3-year follow-up. Leukemia. 2012;26:2197-2203.

16. Politi J, Shah NP. What does a deep molecular response signify? J Clin Oncol. 2014;32:471-474.

17. Hughes TP, Saglio G, Kantarjian HM, et al. Early molecular response predicts outcomes in patients with chronic myeloid leukemia in chronic phase treated with frontline nilotinib or imatinib. Blood. 2014;123:1353-1360.

18. Radich J. Stem cell transplant for chronic myeloid leukemia in the imatinib era. Semin Hematol. 2010;47:354-361.

19. Gratwohl A, Brand R, Apperley J, et al. Allogeneic hematopoietic stem cell transplantation for chronic myeloid leukemia in Europe 2006: transplant activity, long-term data and current results. An analysis by the Chronic Leukemia Working Party of the European Group for Blood and Marrow Transplantation (EBMT). Haematologica. 2006;91:513-521.

20. Oyekunle A, Klyuchnikov E, Ocheni S, et al. Challenges for allogeneic hematopoietic stem cell transplantation in chronic myeloid leukemia in the era of tyrosine kinase inhibitors. Acta Haematol. 2011;126:30-39.

21. Klyuchnikov E, Schafhausen P, Kröger N, et al. Second-generation tyrosine kinase inhibitors in the post-transplant period in patients with chronic myeloid leukemia or Philadelphia-positive acute lymphoblastic leukemia. Acta Haematol. 2009;122:6-10.

22. Andolina JR, Neudorf SM, Corey SJ. How I treat childhood CML. Blood. 2012;119:1821-1830. 
23. de la Fuente J, Baruchel A, Biondi A, et al. Managing children with chronic myeloid leukaemia (CML): recommendations for the management of CML in children and young people up to the age of 18 years. Br J Haematol. Epub June 30, 2014.

24. Ampatzidou M, Papadhimitriou SI, Goussetis E, Panagiotou JP, Papadakis V, Polychronopoulou S. Chronic myeloid leukemia (CML) in children: classical and newer therapeutic approaches. Pediatr Hematol Oncol. 2012;29:389-394.

25. Wayne AS, Macedo C, Szczudlo T, et al. Nilotinib treatment in pediatric patients (pts) with Philadelphia chromosome-positive $(\mathrm{Ph}+)$ leukemia refractory to prior tyrosine kinase inhibitor (TKI) therapy: results from Nilotinib compassionate use program. Blood. 2008;112:4264. Abstract.

26. Novartis Pharmaceuticals. A pharmacokinetic (PK) study of nilotinib in pediatric patients with Philadelphia chromosome-positive $(\mathrm{Ph}+)$ chronic myelogenous leukemia (CML) or acute lymphoblastic leukemia (ALL). Available from: https://clinicaltrials.gov/ct2/show/ NCT01077544?term=NCT01077544. NLM identifier: NCT01077544 Accessed July 24, 2014.

27. Narayanan KR, Bansal D, Walia R, et al. Growth failure in children with chronic myeloid leukemia receiving imatinib is due to disruption of GH/IGF-1 axis. Pediatr Blood Cancer. 2013;60:1148-1153.

28. Ulmer A, Tabea Tauer J, Glauche I, Jung R, Suttorp M. TK inhibitor treatment disrupts growth hormone axis: clinical observations in children with CML and experimental data from a juvenile animal model. Klin Padiatr. 2013;225:120-126.

29. Haouala A, Widmer N, Duchosal MA, Montemurro M, Buclin T, Decosterd LA. Drug interactions with the tyrosine kinase inhibitors imatinib, dasatinib, and nilotinib. Blood. 2011;117:e75-e87.

30. Nicolini FE, Turkina A, Shen ZX, et al. Expanding Nilotinib Access in Clinical Trials (ENACT): an open-label, multicenter study of oral nilotinib in adult patients with imatinib-resistant or imatinib-intolerant Philadelphia chromosome-positive chronic myeloid leukemia in the chronic phase. Cancer. 2012;118:118-126.

31. Tasigna (nilotinib) [Risk Evaluation and Mitigation Strategies (REMS)]. East Hanover, NJ: Novartis Pharmaceuticals; 2010.

32. Fujita K, Sugiyama M, Akiyama Y, Ando Y, Sasaki Y. The smallmolecule tyrosine kinase inhibitor nilotinib is a potent noncompetitive inhibitor of the SN-38 glucuronidation by human UGT1A1. Cancer Chemother Pharmacol. 2011;67:237-241.
33. Cortes JE, Hochhaus A, le Coutre PD, et al. Minimal crossintolerance with nilotinib in patients with chronic myeloid leukemia in chronic or accelerated phase who are intolerant to imatinib. Blood. 2011;117:5600-5606.

34. Alzamora MT, Forés R, Baena-Díez JM, et al. The peripheral arterial disease study (PERART/ARTPER): prevalence and risk factors in the general population. BMC Public Health. 2010;10:38.

35. Kim TD, Rea D, Schwarz M, et al. Peripheral artery occlusive disease in chronic phase chronic myeloid leukemia patients treated with nilotinib or imatinib. Leukemia. 2013;27:1316-1321.

36. Quintás-Cardama A, Kantarjian H, O'Brien S, et al. Pleural effusion in patients with chronic myelogenous leukemia treated with dasatinib after imatinib failure. J Clin Oncol. 2007;25:3908-3914.

37. Gugliotta G, Castagnetti F, Fogli M, Cavo M, Baccarani M, Rosti G. Impact of comorbidities on the treatment of chronic myeloid leukemia with tyrosine-kinase inhibitors. Expert Rev Hematol. 2013;6:563-574

38. Goldman J. Monitoring minimal residual disease in BCR-ABL-positive chronic myeloid leukemia in the imatinib era. Curr Opin Hematol. 2005; 12:33-39.

39. Jabbour E, Kantarjian HM, Jones D, et al. Imatinib mesylate dose escalation is associated with durable responses in patients with chronic myeloid leukemia after cytogenetic failure on standard-dose imatinib therapy. Blood. 2009;113:2154-2160.

40. Apperley JF. Part I: Mechanisms of resistance to imatinib in chronic myeloid leukaemia. Lancet Oncol. 2007;8:1018-1029.

41. National Comprehensive Cancer Network. Chronic Myelogenous Leukemia. Fort Washington (PA): NCCN; 2014.

42. Wu EQ, Johnson S, Beaulieu N. Healthcare resource utilization and costs associated with non-adherence to imatinib treatment in chronic myeloid leukemia patients. Curr Med Res Opin. 2010;26:61-69.

43. Wu EQ, Guerin A, Yu AP, Bollu VK, Guo A, Griffin JD. Retrospective real-world comparison of medical visits, costs, and adherence between nilotinib and dasatinib in chronic myeloid leukemia. Curr Med Res Opin. 2010;26:2861-2869.

44. Pavey T, Hoyle M, Ciani O, et al. Dasatinib, nilotinib and standarddose imatinib for the first-line treatment of chronic myeloid leukaemia: systematic reviews and economic analyses. Health Technol Assess. 2012;16:iii-iv, 1-277.
Blood and Lymphatic Cancer: Targets and Therapy

\section{Publish your work in this journal}

Blood and Lymphatic Cancer: Targets and Therapy is an international, peer-reviewed, open access journal focusing on blood and lymphatic cancer research, identification of therapeutic targets and the optimal use of preventative and integrated treatment interventions to achieve improved outcomes, enhanced survival and quality of life for the

\section{Dovepress}

cancer patient. The manuscript management system is completely online and includes a very quick and fair peer-review system. Visit http://www.dovepress.com/testimonials.php to read real quotes from published authors. 